Check for updates

Cite this: Phys. Chem. Chem. Phys., $2021,23,2320$

Received 4th December 2020 Accepted 11th January 2021

DOI: 10.1039/d0cp06281b

rsc.li/pccp

\section{High field parahydrogen induced polarization of succinate and phospholactate $\dagger$}

\author{
Stephan Berner, (D) *abc Andreas B. Schmidt, (D) *ad Frowin Ellermann, (D) d

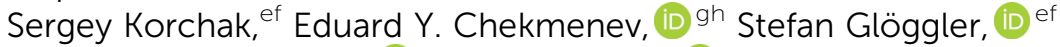 \\ Dominik von Elverfeldt, (D) Jürgen Hennig $\mathbb{D}^{a}$ and Jan-Bernd Hövener (D)
}

\begin{abstract}
The signal enhancement provided by the hyperpolarization of nuclear spins of metabolites is a promising technique for diagnostic magnetic resonance imaging (MRI). To date, most ${ }^{13} \mathrm{C}$-contrast agents are hyperpolarized utilizing a complex or cost-intensive polarizer. Recently, the in situ parahydrogen-induced ${ }^{13} \mathrm{C}$ hyperpolarization was demonstrated. Hydrogenation, spin order transfer (SOT) by a pulsed NMR sequence, in vivo administration, and detection was achieved within the magnet bore of a 7 Tesla MRI system. So far, the hyperpolarization of the xenobiotic molecule $1-{ }^{13} \mathrm{C}$-hydroxyethylpropionate (HEP) and the biomolecule $1{ }^{13} \mathrm{C}$-succinate (SUC) through the PH-INEPT+ sequence and a SOT scheme proposed by Goldman et al., respectively, was shown. Here, we investigate further the hyperpolarization of SUC at 7 Tesla and study the performance of two additional SOT sequences. Moreover, we present first results of the hyperpolarization at high magnetic field of ${ }^{1-}{ }^{13} \mathrm{C}$-phospholactate (PLAC), a derivate to obtain the metabolite lactate, employing the $\mathrm{PH}-$ INEPT+ sequence. For SUC and PLAC, ${ }^{13} \mathrm{C}$ polarizations of about $1-2 \%$ were achieved within seconds and with minimal equipment. Effects that potentially may explain loss of ${ }^{13} \mathrm{C}$ polarization have been identified, i.e. low hydrogenation yield, fast $T_{1} / T_{2}$ relaxation and the rarely considered ${ }^{13} \mathrm{C}$ isotope labeling effect.
\end{abstract}

\section{Introduction}

Magnetic Resonance Imaging (MRI) is a widely used clinical imaging modality due to its excellent soft tissue contrast and non-ionizing radiation. In clinical routine, however, the available signal-to-noise ratio (SNR) is limiting its application to imaging and probing the properties of the abundant protons $\left({ }^{1} \mathrm{H}\right)$ of water and lipids. While this is sufficient for important and essential diagnostics, many other applications such as sodium imaging ${ }^{1}$ or metabolic imaging are not feasible in clinical routine. At room temperature and with currently available magnets, only a few out of a million nuclear spins

\footnotetext{
${ }^{a}$ Department of Radiology, Medical Physics, Medical Center, University of Freiburg, Faculty of Medicine, Germany. E-mail: stephan.berner@uniklinik-freiburg.de, andreas.schmidt@uniklinik-freiburg.de

${ }^{b}$ German Consortium for Cancer Research (DKTK), Germany

${ }^{c}$ German Cancer Research Center (DKFZ), Germany

${ }^{d}$ Department of Radiology and Neuroradiology, Section Biomedical Imaging, MOIN CC, University Medical Center Schleswig-Holstein, Kiel University, Germany ${ }^{e}$ NMR Signal Enhancement Group, Max-Planck-Institute for Biophysical Chemistry, Göttingen, Germany

${ }^{f}$ Center for Biostructural Imaging of Neurodegeneration, Göttingen, Germany ${ }^{g}$ Department of Chemistry, Integrative Biosciences (IBio), Wayne State University, Karmanos Cancer Institute (KCI), Detroit, Michigan 48202, USA

${ }^{h}$ Russian Academy of Sciences, Leninskiy Prospekt 14, Moscow 119991, Russia $\dagger$ Electronic supplementary information (ESI) available. See DOI: 10.1039/ d0cp06281b
}

effectively contribute to the Magnetic Resonance (MR) signal according to the Boltzmann distribution. For ${ }^{1} \mathrm{H}$, the low polarization is compensated by a high concentration in water and lipids. Hence, sufficient SNR is obtained and ${ }^{1} \mathrm{H}$ MRI is feasible. For metabolites or X-nuclei, i.e. a nucleus other than proton, such as ${ }^{23} \mathrm{Na},{ }^{13} \mathrm{C}$ or ${ }^{15} \mathrm{~N}$, the situation is different as their concentration, polarization, or natural abundance is much lower. Hence, in many cases, the MR signals of metabolites and X-nuclei are well below the detection limit and signal averaging is required. ${ }^{1-4}$

The hyperpolarization, i.e. the creation of a non-equilibrium overpopulation of the Zeeman eigenstates, is a method to boost the polarization of MR active nuclei (and thus its signal) by many orders of magnitude. ${ }^{5}$ For instance, hyperpolarized pyruvate, lactate, and fumarate, have been successfully employed to visualize metabolic processes. Cancer was detected and response to treatment was measured in vivo by Magnetic Resonance Spectroscopy Imaging without background signal. ${ }^{6-9}$ Because of the short lifetime of ${ }^{13} \mathrm{C}$ hyperpolarization, the sensitivity-enhanced detection of metabolic conversion is limited to a time-window of rarely more than two minutes. ${ }^{7}$

The most established and commercially available hyperpolarization technique is dissolution Dynamic Nuclear Polarization (d-DNP). ${ }^{10,11}$ However, the method is time-consuming and expensive $\left(10^{6} €\right.$ or more). Typically, achieving a ${ }^{13} \mathrm{C}$ polarization of $P>10 \%$ requires $>30$ min per sample. 
Another hyperpolarization method is based on the spin order of parahydrogen, i.e. dihydrogen $\left(\mathrm{H}_{2}\right)$ enriched in its nuclear singlet state (PHIP). ${ }^{12-14}$ Parahydrogen $\left(p \mathrm{H}_{2}\right)$ has a combined spin of zero, i.e. it is MR invisible. However, its perfectly ordered spin state can be used to achieve X-nuclei hyperpolarization in several ways. By adding $\mathrm{pH}_{2}$ to an unsaturated molecule (hydrogenation), the spin order becomes available in the product molecules' spin system. Under PASADENA ${ }^{13}$ conditions, the ${ }^{1} \mathrm{H}$ spin order can be transferred to ${ }^{13} \mathrm{C}$ through evolution under $J$-coupling and spin manipulations by spin order transfer (SOT) pulse sequences. ${ }^{15-24}$ These sequences consist of periods of free evolution and radiofrequency (RF) pulses applied to ${ }^{1} \mathrm{H}$ and ${ }^{13} \mathrm{C}$.

The $p \mathrm{H}_{2}$ based hyperpolarization procedure is typically executed in an external device (the "polarizer") operating at low magnetic fields in the range of a few milliTesla. ${ }^{25-33}$ At these fields, the ${ }^{13} \mathrm{C}$ hyperpolarization of lactate derivatives and (diethyl-) succinate as high as $15 \%$ and $28 \%$, respectively, have been demonstrated..$^{31,32,34-37}$ The hyperpolarization of lactate is promising, because high lactate concentrations are sustained well in living organisms and lactate may be used for the investigation of brain and energy metabolism. ${ }^{9,38-40}$ Please note that purification of the solution from the catalyst and reaction side product is required prior rigorous in vivo application.

For metabolic imaging of molecules hyperpolarized by low-field PHIP or d-DNP, the samples are typically transferred to a high field detection site. During this transfer, $T_{1}$-relaxation reduces the precious signal enhancement.

Recently, the hyperpolarization of molecules at high field, in an NMR spectrometer or directly in an MRI, has made great progress. ${ }^{13} \mathrm{C}$ polarizations of about $60 \%$ and $9 \%$ for esters of the metabolites acetate and pyruvate, respectively, have been achieved in an NMR spectrometer at $7 \mathrm{~T}^{22,41}$ Moreover, synthesis amid the magnet bore allows a dramatically enhanced nuclear alignment (SAMBADENA) and we demonstrated that high field methods can be employed to conduct a variant of PASADENA in an MRI system. ${ }^{42-44}$ Dispensing with the need of a transfer, we administrated and imaged an aqueous hyperpolarized solution within no more than $15 \mathrm{~s}$ after hyperpolarization.

So far, the SAMBADENA hyperpolarization of the xenobiotic ${ }^{13} \mathrm{C}$ agent $1-{ }^{13} \mathrm{C}, 2,3,3-{ }^{2} \mathrm{H}_{3}$-hydroxyethylpropionate (HEP) and the biomolecule $1-{ }^{13} \mathrm{C}, 2,3-{ }^{2} \mathrm{H}_{2}$-succinate (SUC) was shown. The different molecular structure of the two substrates required the application of two different SOT sequences for efficient polarization transfer. For hyperpolarization of HEP, the SOT sequence PH-INEPT $+{ }^{23}$ was used, whereas for hyperpolarization of SUC, the sequence proposed by Goldman et al. ${ }^{17}$ was employed. In both cases, ${ }^{1} \mathrm{H}$ and ${ }^{13} \mathrm{C}$ RF pulses were played out for spin order transfer. Calculations in simplified models predicted a ${ }^{13} \mathrm{C}$ polarization of $49 \%$ and $99 \%$ for HEP and SUC, respectively. ${ }^{42,44,45}$ Experimentally, reduced ${ }^{13} \mathrm{C}$ polarizations of about $20 \%$ for HEP and $10 \%$ for SUC were found. ${ }^{42,44}$ The low SUC polarization was attributed to singlet-triplet mixing during hydrogenation that led to a 10 -fold reduction of available singlet spin order. ${ }^{44}$

Here, we present initial experiences of the SAMBADENA hyperpolarization of $1{ }^{13} \mathrm{C}-{ }^{2} \mathrm{H}_{2}$-phospholactate (PLAC) at $7 \mathrm{~T}$ in an MRI. Furthermore, we investigate the hyperpolarization of SUC with two other SOT sequences, denoted as $\mathrm{ECHO}^{23,46}$ and ADAPT. ${ }^{19}$ For both SUC and PLAC, we investigated the hydrogenation and SOT efficiency by varying hydrogenation durations and SOT sequence parameters. Moreover, the influence of $\mathrm{pH}$ buffer solutions on the PLAC polarization level was investigated. Quantum mechanical simulations were performed to understand experimental findings better.

\section{Theory and simulations}

\section{${ }^{13} \mathrm{C}$ PASADENA agents and spin order transfer sequences}

Succinate. As the molecule SUC is expected to form an $\mathrm{AA}^{\prime} \mathrm{X}$ spin system to a good approximation, ${ }^{37}$ spin order transfer sequences tailored to strongly coupled protons are well suited (Fig. 1a). $\mathrm{AA}^{\prime} \mathrm{X}$ spin systems consist of two chemically equivalent but magnetically inequivalent protons $A$ and $\mathrm{A}^{\prime}$. The values of the $J$-couplings were taken from literature as $J_{12}=7.41 \mathrm{~Hz}$, $J_{13}=-7.15 \mathrm{~Hz}$, and $J_{23}=5.82 \mathrm{~Hz} .{ }^{37}$ The isotropic chemical shifts of the two ${ }^{1} \mathrm{H}$ nuclei and ${ }^{13} \mathrm{C}$ nucleus were determined by NMR spectroscopy and take the values $\delta_{1}\left({ }^{1} \mathrm{H}\right) \approx \delta_{2}\left({ }^{1} \mathrm{H}\right) \approx 2.75 \mathrm{ppm}$, and $\delta_{3}\left({ }^{13} \mathrm{C}\right)=176 \mathrm{ppm}$. The two protons experience a slightly different effective magnetic field because of isotopic ${ }^{13} \mathrm{C}$ labeling and become chemically inequivalent, i.e. $\delta_{1}\left({ }^{1} \mathrm{H}\right) \neq \delta_{2}\left({ }^{1} \mathrm{H}\right)$. Simulations and experiments indicate that the actual difference of the Larmor frequencies at $7 \mathrm{~T}$ is about $4 \mathrm{~Hz}$ (Fig. 5 and ESI $\dagger$ ). To our knowledge, this effect was not taken into account in simulations of SOT before. Please note that couplings to ${ }^{2} \mathrm{H}$ spins are not considered as the ${ }^{2} \mathrm{H}-{ }^{1} \mathrm{H}$ interactions are a factor of $\gamma\left({ }^{1} \mathrm{H}\right) / \gamma\left({ }^{2} \mathrm{H}\right)=6$ smaller than the ${ }^{1} \mathrm{H}-{ }^{1} \mathrm{H}$ couplings. ${ }^{47} \mathrm{SUC}$ is an intermediate in the tricarboxylic acid cycle and may be used for the diagnosis of brain cancer and potentially other diseases. ${ }^{35,36,48}$

Here, two SOT sequences, denoted as $\mathrm{ADAPT}^{19}$ and $\mathrm{ECHO},{ }^{23,46}$ tailored to chemically equivalent protons were employed for hyperpolarization of SUC (Fig. 1). In contrast to the recently reported hyperpolarization of SUC with Goldman's sequence, ${ }^{17,44,45}$ ECHO and ADAPT require ${ }^{13} \mathrm{C}$ RF pulses only and less RF excitations. Hence, ECHO and ADAPT are expected to be less susceptible to erroneous flip angles and off-resonance effects than Goldman's sequence. In addition, ECHO does not require heteronuclear decoupling during hydrogenation. This is of major importance for MR systems with limited power specifications of the transmit array.

The ADAPT sequence ${ }^{19}$ converts singlet spin order $\mathbf{I}_{1} \mathbf{I}_{2}=$ $\mathbf{I}_{1 z} \mathbf{I}_{2 z}+\mathbf{I}_{1 y} \mathbf{I}_{2 y}+\mathbf{I}_{1 x} \mathbf{I}_{2 x}$ into detectable ${ }^{13} \mathrm{C}$ magnetization (Fig. 1b). Therefore, heteronuclear decoupling is essential during hydrogenation to preserve singlet state in the product system. Depending on the flip angle $\alpha_{x} \in\left(0^{\circ}, 180^{\circ}\right)$, the free evolution interval $t_{\mathrm{AD}, \alpha}$ and number of ADAPT cycles $n_{\alpha}$ have to be set appropriately. In this contribution, the parameters were chosen as $\alpha_{x}=90^{\circ}$ and $n_{\alpha}=2$. Please note that according to the original description of the ADAPT sequence, ${ }^{19}$ no $180^{\circ}$ spin echo pulses are played out during free evolution. Another sequence, denoted as ECHO here, transfers $\left(\mathbf{I}_{1 z}-\mathbf{I}_{2 z}\right) \mathbf{I}_{3 z}{ }^{1} \mathrm{H}$ spin order into ${ }^{13} \mathrm{C}$ polarization and requires neither decoupling during 
a) Hydrogenation and J-couplings

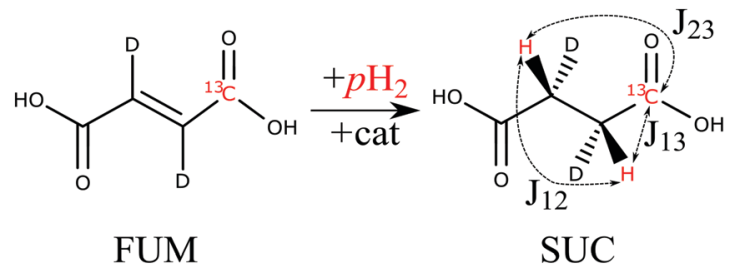

b) ADAPT

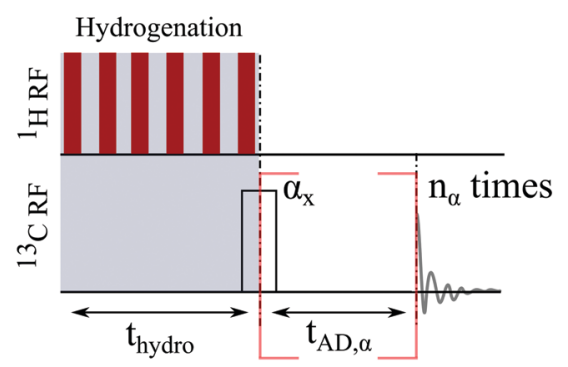

c) $\mathrm{ECHO}$

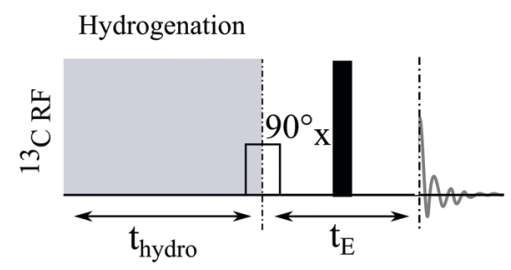

Fig. 1 Hydrogenation reaction, J-coupling network, and SOT sequences for succinate and strongly coupled protons. (a) The $\mathrm{pH}_{2}$ protons are added to fumarate (FUM) via a rhodium-based catalyst at $90{ }^{\circ} \mathrm{C}$ and 20 bar, forming succinate (SUC). The values of the $J$-couplings read $J_{12}=7.41 \mathrm{~Hz}$, $J_{13}=-7.15 \mathrm{~Hz}$, and $J_{23}=5.82 \mathrm{~Hz}$. The SOT sequences ADAPT ${ }^{19}(\mathrm{~b})$ and $\mathrm{ECHO}^{23,46}$ (c) were used to transfer the ${ }^{1} \mathrm{H}$ para order to ${ }^{13} \mathrm{C}$ polarization and consist of RF pulses and free evolution times $t_{\mathrm{AD}, \alpha}$ and $t_{\mathrm{E}}$. Note that ADAPT requires heteronuclear decoupling during hydrogenation to preserve singlet state (red bars), whereas ECHO does not. The black bar denotes a $180^{\circ}$ spin echo pulse along the $x$ - or $y$-axis at $t_{E} / 2$.

hydrogenation nor ${ }^{1} \mathrm{H}$ RF pulses (Fig. 1c). After hydrogenation, a $90^{\circ}{ }^{13} \mathrm{C}$ RF pulse followed by free evolution and a $180^{\circ}$ spin echo pulse is played out.

Phospholactate. The molecule PLAC forms an ABDX 4-spin system at $7 \mathrm{~T}$ with two chemically inequivalent ${ }^{1} \mathrm{H}$ spins $\mathrm{A}$ and $\mathrm{B}$, one ${ }^{31} \mathrm{P}$ spin $\mathrm{D}$, and one ${ }^{13} \mathrm{C}$ spin $\mathrm{X}$. The chemical shift difference of the two protons, $225 \mathrm{~Hz}$ at $7 \mathrm{~T}$, is much larger than the mutual $J$-coupling of $6.90 \mathrm{~Hz}$ (Fig. 2). The values of the chemical shifts $\delta_{i}$ in ppm and $J$-couplings $J_{i j}$ in $\mathrm{Hz}$ were taken from literature ${ }^{31}$ and read $\delta_{1}\left({ }^{1} \mathrm{H}\right)=1.29, \delta_{2}\left({ }^{2} \mathrm{H}\right)=4.33, \delta_{3}\left({ }^{13} \mathrm{C}\right)=$ $182.1, \delta_{4}\left({ }^{31} \mathrm{P}\right)=4.53, J_{12}=6.90, J_{13}=4.07, J_{23}=3.80, J_{34}=6.52$, and $J_{24}=8.60$. The sequence PH-INEPT ${ }^{23}$ was employed for hyperpolarization as it was designed for systems containing weakly coupled protons. Originally, it was assumed that the singlet spin order $\mathbf{I}_{1} \mathbf{I}_{2}$ of $p \mathbf{H}_{2}$ is converted into $\mathbf{I}_{1 z} \mathbf{I}_{2 z}$ spin order by averaging of the transversal $\mathbf{I}_{1 y} \mathbf{I}_{2 y}$-and $\mathbf{I}_{1 x} \mathbf{I}_{2 x}$-components during hydrogenation at high field. ${ }^{23}{ }^{1} \mathrm{H}$ and ${ }^{13} \mathrm{C} 180^{\circ}$ spin a) Hydrogenation and J-couplings<smiles>[2H]C([2H])=C(OP(=O)(O)O)C(=O)O</smiles>

PEP

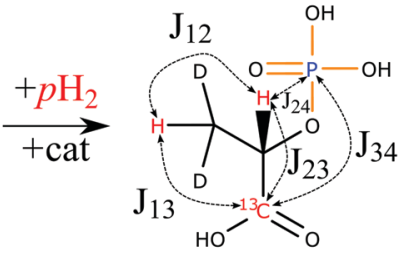

PLAC

\section{b) PH-INEPT+}

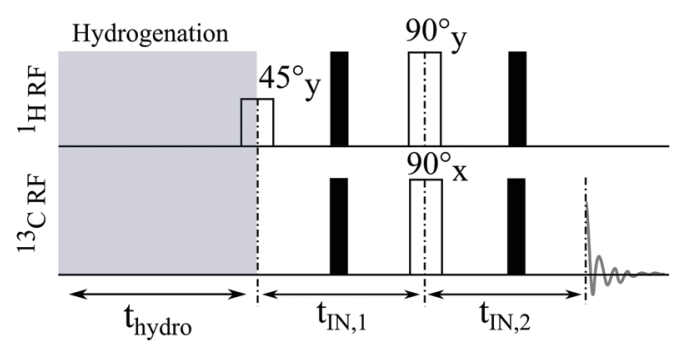

Fig. 2 Hydrogenation reaction, J-coupling network, and PH-INEPT+ sequence used for PLAC. By addition of $\mathrm{pH}_{2}$, phosphoenolpyruvate (PEP) is converted to PLAC (a). By means of the PH-INEPT+ pulse sequence, ${ }^{23}$ spin order is transferred to ${ }^{13} \mathrm{C}$ (b). In a simplified model, PLAC is a 4-spin system composed of two weakly coupled protons, one ${ }^{13} \mathrm{C}$ nucleus, and one ${ }^{31} \mathrm{P}$ nucleus (blue) with the corresponding $\mathrm{J}$-couplings $J_{12}=6.90 \mathrm{~Hz}$, $J_{13}=4.07 \mathrm{~Hz}, J_{23}=3.80 \mathrm{~Hz}, J_{34}=6.52 \mathrm{~Hz}$, and $J_{24}=8.60 \mathrm{~Hz}$. ${ }^{31}$ The sequence consists of two free evolution intervals and ${ }^{1} \mathrm{H} /{ }^{13} \mathrm{C}$ RF pulses. Black bars denote $180^{\circ}$ spin echo pulses.

echo pulses are played out in between the other pulses to compensate for different precession frequencies.

PLAC was previously hyperpolarized in low magnetic fields and a maximum polarization of up to $15 \%$ was achieved inside a low-field polarizer. ${ }^{28,31,32,34,49}$ It was reported that the phosphate group in PLAC (Fig. 2a, orange) is removed by blood dephosphorylation quickly after in vivo administration such that the metabolite $1{ }^{13} \mathrm{C}$-lactate is obtained. ${ }^{49}$ Please note, that for now, the PHIP-SAH strategy proposed by Reineri et al. is the only method by which $p \mathrm{H}_{2}$ hyperpolarized $1{ }^{1-}{ }^{13} \mathrm{C}$-lactate has been obtained..$^{50,51}$

\section{Spin Hamiltonian, density matrix, and strategy for SOT computations}

Spin Hamiltonian. The spin system was driven by the Zeeman interaction considering the isotropic chemical shift $\left(\delta_{i}\right)$ and $J$-couplings $\left(J_{i j}\right)$. The Hamiltonian of PLAC in the laboratory frame of reference reads $(\hbar=1)$

$$
\mathcal{H}_{\mathrm{PLAC}}=-B_{0} \sum_{i} \gamma_{i}\left(1+\delta_{i}\right) \mathbf{I}_{i z}+2 \pi \sum_{i<j} J_{i j} \mathbf{I}_{i} \mathbf{I}_{j}
$$

where the summation runs over all spins and all coupling pairs with $I_{i} I_{j}=I_{i x} I_{j x}+I_{i y} I_{j y}+I_{i z} I_{j z}$. Here, $\gamma_{i}$ denotes the gyromagnetic ratios of ${ }^{1} \mathrm{H},{ }^{13} \mathrm{C}$, and ${ }^{31} \mathrm{P}\left(\gamma_{1}=\gamma_{2}=\gamma_{\mathrm{H}}, \gamma_{3}=\gamma_{\mathrm{C}}\right.$, and $\left.\gamma_{4}=\gamma_{\mathrm{P}}\right)$. 
The Hamiltonian of SUC is of the form

$$
\begin{aligned}
\mathcal{H}_{\mathrm{SUC}}= & -B_{0}\left[\gamma_{\mathrm{H}}\left(1+\delta_{1}\right)\left(\mathbf{I}_{1 z}+\mathbf{I}_{2 z}\right)+\gamma_{\mathrm{C}}\left(1+\delta_{3}\right) \mathbf{I}_{3 z}\right]+\Delta_{\mathrm{CS}} \mathbf{I}_{2 z} \\
& +2 \pi \sum_{i<j} J_{i j} \mathbf{I}_{i} \mathbf{I}_{j}
\end{aligned}
$$

The factor $\Delta_{\mathrm{CS}}$ was introduced to account for a difference of the ${ }^{1} \mathrm{H}$ Larmor frequencies induced by the ${ }^{13} \mathrm{C}$ labeling and consequential symmetry breaking. ${ }^{52}$

Density matrix of PLAC and SUC. The density matrices representing the spin system after hydrogenation were obtained by numerical simulations. As the reaction takes place over a finite period of time $t_{\mathrm{hydr}}$, each hydrogenated molecule experiences a different evolution time $t$. This condition is approximated by calculating the time-averaged density operator

$$
\overline{\boldsymbol{\rho}_{0}}=\frac{\sum_{t=n \cdot \Delta t}^{t_{\text {hydr }}} p(t) \mathbf{Q}(t) \rho_{0} \mathbf{Q}(t)^{\dagger}}{\sum_{t=n \cdot \Delta t}^{t_{\text {hydr }}} p(t)}
$$

where $p(t)$ is a weighting function that reflects the amount of hydrogenated molecules at different points in time. $\mathbf{Q}$ is an operator that either describes free evolution (ECHO and $\mathrm{PH}$ INEPT+, Fig. 1 and 2) or heteronuclear MLEV16 decoupling ${ }^{53}$ (ADAPT, Fig. 1b). The free evolution operator is given by the matrix exponential of $-i \mathcal{H} t$. The exponential was computed numerically employing the MatLab function “expm”. Ideal RF pulses (on-resonant and instantaneous rotations) are approximated by the exponential of $-i \alpha \mathbf{I}_{j k}$ where $\alpha$ denotes the flip angle and $\mathbf{I}_{j k}$ represents the spin operator of $\operatorname{spin} j$ in $k$-direction $(k=x, y$, or $z$ ). MLEV16 decoupling pulses of finite duration $t_{\mathrm{MLEV}}$ were approximated by a single rotation with subsequent time evolution $t_{\mathrm{MLEV}}$. The pulse duration of a single $90^{\circ}$ excitation within the MLEV16 pulse train was chosen as $t_{\mathrm{MLEV}}\left(90^{\circ}\right) \approx$ $1 \mathrm{~ms}$ in order to simulate the experimental conditions (Table 1).

When simulating the hydrogenation process of PLAC numerically, the sudden addition of $\mathrm{pH}_{2}$ was assumed, resulting in a density matrix of the form

$$
\boldsymbol{\rho}_{0}=|\mathrm{S}\rangle\langle\mathrm{S}| \otimes \boldsymbol{\rho}_{{ }^{13} \mathrm{C}} \otimes \boldsymbol{\rho}{ }^{1} \mathrm{P}
$$

The first factor represents singlet state of the two protons, i.e.

$$
|\mathrm{S}\rangle=\frac{1}{\sqrt{2}}(|\alpha \beta\rangle-|\beta \alpha\rangle)
$$

in Zeeman basis. The density matrices of the target nucleus $\rho^{{ }_{13} \mathrm{C}}$ and ${ }^{31} \mathrm{P}$ spin $\rho_{31} \mathrm{P}$ were assumed to be a normalized $2 \times 2$ unity matrix, i.e. non-polarized, neglecting the low thermal ${ }^{13} \mathrm{C}$ and ${ }^{31} \mathrm{P}$ polarization.

For SUC, two different initial density matrices were considered. In an idealized model the density matrix of a single SUCmolecule was given by

$$
\boldsymbol{\rho}_{0}=|S\rangle\langle S| \otimes \rho^{13} \mathrm{C}
$$

However, in previous experiments conducted at $7 \mathrm{~T}$ using the same hydrogenation catalyst as used in this work, singlet- triplet mixing during hydrogenation led to a significant decrease of singlet spin order. ${ }^{44}$ Analysis of ${ }^{1} \mathrm{H}$ PASADENA spectra revealed that the amount of singlet and triplet $\left|T_{0}\right\rangle=$ $\frac{1}{\sqrt{2}}(|\alpha \beta\rangle+|\beta \alpha\rangle)$ spin order was about $55 \%$ and $45 \%$, respectively. Hence, the density matrix $\boldsymbol{\rho}_{0}$ of a single SUC-molecule directly after hydrogenation with $\mathrm{pH}_{2}$ was further assumed to be of the form

$$
\boldsymbol{\rho}_{0}=\left[0.55|S\rangle\left\langle S|+0.45| T_{0}\right\rangle\left\langle T_{0}\right|\right] \otimes \rho_{13} \mathrm{C}
$$

Please note that analytical equations of the time-averaged density matrix were derived for $\mathrm{AB}$ and $\mathrm{AA}$ 'X spin systems by Hübler et al. and Natterer et al. ${ }^{54,55}$ In the latter paper, however, a potential ${ }^{13} \mathrm{C}$ isotope effect was neglected.

Computations of SOT sequences. To simulate the effect of free evolution and RF pulses on ${ }^{1} \mathrm{H}$ and ${ }^{13} \mathrm{C}$, rotation and time evolution operators were applied to the time-averaged density matrix $\overline{\boldsymbol{\rho}_{0}}$. For different initial states of the spin system (eqn (4)(7)) and different values of $\Delta_{\mathrm{CS}}$ (eqn (2)), the ${ }^{13} \mathrm{C}$ polarization yield was calculated as a function of the free evolution intervals of the SOT sequences ( $0.05 \mathrm{~ms}$ time increments). Moreover, the influence of the ${ }^{31} \mathrm{P}$ spin in PLAC was investigated as computations were conducted excluding or including the ${ }^{31} \mathrm{P}$ spin. The transversal polarization was determined by taking the trace over the matrix product of the final density matrix (after application of the SOT sequence) and the polarization operator $2\left(\mathbf{I}_{3 x}+\mathbf{i I}_{3 y}\right)$. Relaxation effects, for instance due to dipole-dipole interaction or chemical exchange in hydroxyl groups, were neglected in all simulations.

\section{Two-site kinetic model of the hydrogenation reaction}

To estimate the reaction kinetics and relaxation of ${ }^{1} \mathrm{H}$ spin order during hydrogenation experimentally, the ${ }^{13} \mathrm{C}$ polarization was measured as a function of the total hydrogenation duration $t_{\text {hydr }}$. A model was developed to fit the data (eqn (8)), consisting of an asymptotical increase of the hydrogenated molecules $\left(\propto 1-\exp \left[-t_{\text {hydr }} / t_{\text {cat }}\right]\right)$, and a mono-exponential decay of the polarization due to relaxation $\left(\propto \exp \left[-t_{\text {hydr }} /\right.\right.$ $t_{\text {spin }} .^{42,56}$

$$
P\left(t_{\text {hydr }}\right)=\frac{P_{\text {max }}}{\frac{t_{\text {cat }}}{t_{\text {spin }}}-1}\left[\mathrm{e}^{\frac{-\left(t_{\text {hydr }}-t_{0}\right)}{t_{\text {cat }}}}-\mathrm{e}^{\frac{-\left(t_{\text {hydr }}-t_{0}\right)}{t_{\text {spin }}}}\right]
$$

The coefficients $P_{\max }$ and $t_{0}$ represent fit parameters that allow the maximum polarization to be adjusted and to consider an activation delay between application of $\mathrm{pH}_{2}$ pressure and the onset of the hydrogenation reaction, respectively. The time constants for hydrogenation and relaxation of the initial ${ }^{1} \mathrm{H}$ spin order are denoted by $t_{\text {cat }}$ and $t_{\text {spin }}$, respectively. 


\section{Experiments}

\section{Enrichment of parahydrogen}

The fraction of $p \mathrm{H}_{2}$ was enriched to $\approx 90 \%$ using a custom-built $\mathrm{pH}_{2}$ converter at a temperature of $21 \mathrm{~K}$ as described previously. ${ }^{42-44,57}$ The $p \mathrm{H}_{2}$-enriched gas was stored in $2.7 \mathrm{~L}$ steel or $0.5 \mathrm{~L}$ aluminum reservoirs at a pressure of 50 bar and was used within 10 hours.

\section{Sample preparation}

$1-{ }^{13} \mathrm{C}, 2,3-{ }^{2} \mathrm{H}_{2}$-succinate was formed by hydrogenation of $1{ }^{13} \mathrm{C}, 2,3-{ }^{2} \mathrm{H}_{2}$-fumarate (FUM, $118.08 \mathrm{~g} \mathrm{~mol}^{-1}$ molecular weight, CAS: 24461-32-3, Cambridge Isotope Laboratories, deionized $\mathrm{H}_{2} \mathrm{O}$ as solvent).

By hydrogenation of the precursor $1-{ }^{13} \mathrm{C}-{ }^{2} \mathrm{H}_{2}$-phosphoenolpyruvate (PEP, $170.0 \mathrm{~g} \mathrm{~mol}^{-1}$, in-house synthesis as described in literature, ${ }^{32} \mathrm{D}_{2} \mathrm{O}$ as solvent) PLAC is obtained.

For hyperpolarization experiments, reaction solutions were prepared with different concentrations of substrate $\left(c_{\mathrm{FUM}}=5 \mathrm{mM}\right.$, $\left.c_{\mathrm{PEP}}=10 \mathrm{mM}\right)$ and catalyst $\left(c_{\text {cat }} \approx 2 \mathrm{mM}\right.$ ) using a Schlenk line and degassing as described previously. ${ }^{42-44}$ The catalyst was obtained by mixing 1,4-bis-[(phenyl-3-propane sulfonate) phosphine]butane disodium salt ( $\approx 2.2 \mathrm{mM}$, Q36333, $562.5 \mathrm{~g} \mathrm{~mol}^{-1}$, Sigma Aldrich) with bis(norbornadiene)rhodium(I) tetrafluoroborate $(\approx 2.0 \mathrm{mM}$, CAS: 36620-11-8, StremChemicals).

Previous reports of $p \mathrm{H}_{2}$-induced polarization of SUC and PLAC suggested adjusting the $\mathrm{pH}$ value to either low (3-4) or high (10-11) $\mathrm{pH}$ for well-resolved $J$-couplings, to reduce relaxation due to chemical exchange, and to achieve a high hydrogenation yield. ${ }^{32,37}$ Hence, different substances B.1-B.3 were used to buffer the reaction solution to a pH of 3 for SUC and about 10 for PLAC.

B.1. A pH buffer based on potassium dihydrogen phosphate $\left(\mathrm{KH}_{2} \mathrm{PO}_{4}\right.$, CAS: 7778-77-0) and phosphoric acid $\left(\mathrm{H}_{3} \mathrm{PO}_{4}, \mathrm{CAS}: 7664-\right.$ $38-2)$ for experiments with SUC was prepared $\left(c_{\mathrm{KH}_{2} \mathrm{PO}_{4}}=40 \mathrm{mM}\right.$, $\left.c_{\mathrm{H}_{3} \mathrm{PO}_{4}}=3 \mathrm{mM}\right)$. A pH value of 2.9 was measured at room temperature and before hydrogenation (HI 83141, Hanna Instruments).

B.2. For hyperpolarization of PLAC, sodium phosphate in a powdery form was added to the precursor solution until a $\mathrm{pH}$ of 10.6 was measured $\left(\mathrm{Na}_{3} \mathrm{PO}_{4}\right.$, CAS: 7601-54-9, Sigma Aldrich). The final $\mathrm{Na}_{3} \mathrm{PO}_{4}$ concentration was $\approx 22 \mathrm{mM}$.

B.3. A commercially available stock buffer solution based on boric acid, potassium chloride, and sodium hydroxide with a $\mathrm{pH}$ value of 10.0 at $20{ }^{\circ} \mathrm{C}$ was used for experiments with PLAC (material number 9438, Merck).

\section{Experimental setup, workflow, and performed experiments}

As the experimental setup, workflow, and hydrogenation reactor were described in detail in several previous reports, ${ }^{42-44}$ only a brief overview is provided here. The experimental setup consists of a $7 \mathrm{~T}$ preclinical MRI system (Biospec 70/20, Bruker) and a linearly polarized ${ }^{1} \mathrm{H}^{13}{ }^{13}$ volume coil (Rapid Biomed). The hyperpolarization experiments were conducted with the help of two or three electromagnetic valves $V$ (type 124, Bürkert) that were controlled with a custom written software (MatLab, National Instruments, Fig. 3). To start the experiment, gaseous

\section{a) Setup 1}

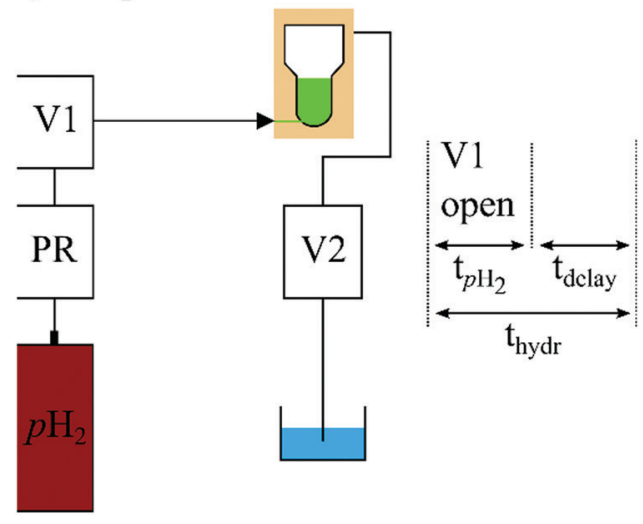

b) Setup 2

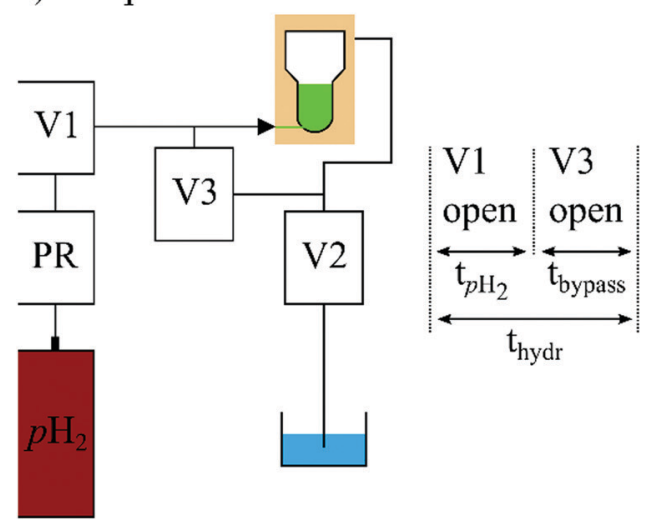

Fig. 3 Experimental setups and timetables showing the actuation of the magnetic valves. Setup 1: the $\mathrm{pH}_{2}$ reservoir was connected to the inlet of the reaction chamber via a pressure regulator (PR), valve $\mathrm{V} 1$, and a nonmetallic check valve. $\mathrm{V} 1$ was opened for a period of $t_{p \mathrm{H}_{2}}$ to initialize the chemical reaction. The SOT sequence was executed after an additional delay $\left(t_{\text {delay }}\right)$, resulting in a total hydrogenation time $t_{\text {hydr }}$. The outlet valve V2 was closed during the entire hydrogenation and hyperpolarization process. After signal detection, V2 was opened and the solution was flushed out from the reactor and caught in a water bath (blue). Setup 2 was identical to setup 1 but an additional valve (V3) was installed to bypass the reactor for equilibration of the pressure between in- and outlet. This valve was opened after $t_{\mathrm{pH}_{2}}$ for a period of $t_{\text {bypass }}$ to stop the $\mathrm{pH}_{2}$ injection and to prevent the formation of gas bubbles. When heteronuclear decoupling was required, decoupling was played out only during $t_{\text {bypass. }}$

$p \mathrm{H}_{2}$ at a pressure of 20 bar was injected into a reactor filled with substrate solution through an inlet at the bottom of the reactor. After the experiment and data acquisition, pressure was released through an outlet at the top. Prior to experiments, the reactor was heated in a $60{ }^{\circ} \mathrm{C}$ or $90{ }^{\circ} \mathrm{C}$ water bath.

The MRI system was adjusted by placing the reactor filled with $1 \mathrm{~mL}$ of deionized $\mathrm{H}_{2} \mathrm{O}$ in the isocenter of the MRI. The RF coil was tuned and matched manually. The field homogeneity was improved by an automatic first order shimming routine provided by the manufacturer. For experiments with SUC, the ${ }^{1} \mathrm{H}$ center frequency was set to the resonance frequency of the SUC-protons at $\approx 2.8 \mathrm{ppm}$. For hyperpolarization of PLAC, the ${ }^{1} \mathrm{H}$ frequency was centered between the two resonances of 
the $\mathrm{pH}_{2}$-nascent protons at 4.33 and $1.29 \mathrm{ppm}$, i.e. at $\approx 2.8 \mathrm{ppm}$. The durations of the ${ }^{1} \mathrm{H}$ and ${ }^{13} \mathrm{C}$ SOT RF pulses were chosen as $0.5 \mathrm{~ms}$ in all experiments. When heteronuclear decoupling during hydrogenation was required, the ${ }^{1} \mathrm{H}$ MLEV16 decoupling scheme was applied. The pulse length of a single $90^{\circ}{ }^{1} \mathrm{H}$ RF pulse of the MLEV16 sequence was set to $1 \mathrm{~ms}$.

After hyperpolarization experiments, the ${ }^{13} \mathrm{C}$ polarizations were quantified by comparing the hyperpolarized ${ }^{13} \mathrm{C}$ signals with a thermally polarized acetone reference sample $(4 \mathrm{~mL}, 13.5 \mathrm{M}, 1.1 \%$ ${ }^{13} \mathrm{C}$, 50 averages, $90^{\circ}$ excitation, numerical integration with TopSpin, Bruker) assuming a full conversion to SUC and PLAC.

For SUC and ECHO (Fig. 1), the hydrogenation reaction was initialized by injecting pressurized $p \mathrm{H}_{2}$ gas into the reaction chamber by opening the $p \mathrm{H}_{2}$ valve (V1) for time duration $t_{p \mathrm{H}_{2}}=2 \mathrm{~s}$ (Fig. 3, setup 1). After an additional delay of $t_{\text {delay, }}$ i.e. a total hydrogenation duration $t_{\mathrm{hydr}}=t_{p \mathrm{H}_{2}}+t_{\text {delay, }}$, spin order was transferred.

For SUC/ADAPT and PLAC/PH-INEPT+ (Fig. 1 and 2), another setup and workflow was used. This was of particular importance when employing the ADAPT sequence: as previously reported, ${ }^{44}$ heteronuclear decoupling is strongly disturbed during $p \mathrm{H}_{2}$ injection. Distortions of the field homogeneity because of susceptibility gradients induced by the gas bubbles in aqueous solution during $t_{p \mathrm{H}_{2}}$ lead to erroneous RF excitations. Therefore, ${ }^{1} \mathrm{H}$ MLEV16 decoupling pulses were only applied during $t_{\text {bypass }}$ using setup 2 . Thus, the total hydrogenation duration was $t_{\mathrm{hydr}}=t_{p \mathrm{H}_{2}}+t_{\text {bypass }}$.

A series of different hyperpolarization experiments were performed, where the hydrogenation duration, i.e. $t_{\text {delay }}$, or free

Table 1 Performed experiments with SUC at $7 \mathrm{~T}$ and $\mathrm{pH} 2.9$ using buffer B.1. The hyperpolarization was investigated in three experimental series: In SUC.E1, ECHO was applied and the polarization was measured as a function of the hydrogenation duration by variation of $t_{\text {delay }}$ with a fixed free evolution interval. In SUC.E2, the free evolution interval $t_{E}$ was varied while $t_{\text {delay }}$ was fixed. To investigate the polarization scheme of the ADAPT sequence, the free evolution interval $t_{A D, 90^{\circ}}$ was varied with fixed hydrogenation duration $t_{\text {hydr }}=5 \mathrm{~s}$ (SUC.E3)

\begin{tabular}{lll}
\hline & SOT & Timings/parameters \\
\hline SUC.E1 & ECHO & $t_{p \mathrm{H}_{2}}=2 \mathrm{~s}$, variation of $t_{\text {delay }}$ \\
& & $t_{\mathrm{E}}=70.0 \mathrm{~ms}$ \\
& Setup $1,90{ }^{\circ} \mathrm{C}$ \\
SUC.E2 & $t_{p \mathrm{H}_{2}}=2 \mathrm{~s}, t_{\text {delay }}=3 \mathrm{~s}$ \\
& ECHO & Variation of $t_{\mathrm{E}}$ \\
& Setup $1,90{ }^{\circ} \mathrm{C}$ \\
SUC.E3 & $t_{p \mathrm{H}_{2}}=2 \mathrm{~s}, t_{\text {bypass }}=3 \mathrm{~s}$ \\
& & $\alpha_{x}=90^{\circ}, n_{\alpha}=2$ \\
& & Variation of $t_{\mathrm{AD}, 90^{\circ}}$ \\
& & Setup $2,90{ }^{\circ} \mathrm{C}$ \\
& & $t_{\mathrm{MLEV}}\left(90^{\circ}\right)=1 \mathrm{~ms}$
\end{tabular}

evolution intervals, i.e. $t_{\mathrm{IN}, 1 / 2}, t_{\mathrm{E}}, t_{\mathrm{AD}, \alpha}$, were varied (Tables 1 and 2). Furthermore, the influence of different $\mathrm{pH}$ buffer materials on the PLAC polarization was investigated. The ${ }^{1} \mathrm{H} /{ }^{13} \mathrm{C}$ relaxation times and the hydrogenation yield were determined by NMR spectroscopy after hyperpolarization experiments on a 7 T NMR spectrometer (see ESI $\dagger$ ).

\section{Results}

\section{Hyperpolarization of succinate}

Optimization of the hydrogenation duration. SUC was successfully polarized using both ECHO and ADAPT, however, to a lesser degree compared to the results obtained with Goldman's sequence. ${ }^{44}$ When SUC was polarized with different hydrogenation durations $t_{\text {hydr }}$ (SUC.E1), an optimum was found between $3 \mathrm{~s}$ and $6 \mathrm{~s}$ (Fig. 4). By fitting eqn (8) to the data, the relaxation time of $\mathrm{pH}_{2}$-nascent spin order $t_{\text {spin }}$ and the hydrogenation constant $t_{\text {cat }}$ were determined as $t_{\text {spin }}=(13 \pm 5) \mathrm{s}$ and $t_{\text {cat }}=(1.4 \pm$ $0.9) \mathrm{s}$, respectively. This implies that after $5 \mathrm{~s}$ of hydrogenation about $(97 \pm 6) \%$ of all FUM molecules were hydrogenated. Examination of the hydrogenation kinetics by ${ }^{1} \mathrm{H}$ PASADENA/ ALTADENA experiments was not feasible on the MRI system because the ${ }^{1} \mathrm{H}$ resonances were not resolved due to poor field homogeneity and baseline of the MR spectra.

Performance of the ECHO sequence. Computations assuming pure time-averaged singlet state after hydrogenation and chemically equivalent protons, i.e. $100 \%|S\rangle$ before time-averaging and $\Delta_{\mathrm{CS}}=0 \mathrm{~Hz}$ (eqn (2), (3) and (6)), predicted a maximum polarization of $P=48.0 \%$ for $t_{\mathrm{E}}=67.7 \mathrm{~ms}$. This is a quite promising result given that the sequence is simple and short. However, when singlettriplet mixing was taken into account in computations (eqn (7)), a 10-fold decrease in polarization was observed $\left(P=4.8 \%\right.$ for $t_{\mathrm{E}}=$ $67.7 \mathrm{~ms}$ ). Considering the ${ }^{13} \mathrm{C}$ isotope effect with $\Delta_{\mathrm{CS}}=4 \mathrm{~Hz}$ did not lead to significantly different values for $t_{\mathrm{E}} \leq 100 \mathrm{~ms}$. However, less polarization was found for $t_{\mathrm{E}}$ greater than $100 \mathrm{~ms}$ (Fig. 5, red and blue lines).

To verify the simulations, experiments with varying evolution intervals $t_{\mathrm{E}}$ were conducted. Experimentally, a maximum polarization of $P=(0.97 \pm 0.15) \%$ was found for $t_{\mathrm{E}}=60.0 \mathrm{~ms}$ and $70.0 \mathrm{~ms}$ after a $t_{\text {hydr }}=5 \mathrm{~s}$ hydrogenation (SUC.E2, Fig. 5, squares).

The experimental data was similar to the simulations without isotope effect $\left(\Delta_{\mathrm{CS}}=0 \mathrm{~Hz}\right)$, with singlet-triplet mixing, in the range of $t_{\mathrm{E}} \leq 110 \mathrm{~ms}$ and $t_{\mathrm{E}}=190 \mathrm{~ms}$, except for an overall constant scaling factor of 4.94. Significant deviations were

Table 2 Performed experiments with PLAC using setup 2 and PH-INEPT+. First, the hydrogenation conditions, i.e. hydrogenation duration and temperature, were optimized (PLAC.E1 and PLAC.E2). The free evolution intervals $t_{\mathrm{IN}, 1}$ and $t_{\mathrm{IN}, 2}$ of the sequence were varied in another experiment PLAC.E3 with a fixed hydrogenation duration $t_{\text {hydr }}=5 \mathrm{~s}$. The influence of the buffer solutions B. 2 and B. 3 was examined in an additional set of experiments (PLAC.E4)

\begin{tabular}{lll}
\hline & Buffer & Timings/parameters \\
\hline PLAC.E1 & B.2 & $t_{p \mathrm{H}_{2}}=2 \mathrm{~s}$, variation of $t_{\mathrm{bypass}}, t_{\mathrm{IN}, 1}=60.0 \mathrm{~ms}, t_{\mathrm{IN}, 2}=50.0 \mathrm{~ms}, 60^{\circ} \mathrm{C}$ \\
PLAC.E2 & B.2 & $t_{p \mathrm{H}_{2}}=2 \mathrm{~s}$, variation of $t_{\mathrm{bypass}}, \mathrm{t}_{\mathrm{IN}, 1}=60.0 \mathrm{~ms}, t_{\mathrm{IN}, 2}=70.0 \mathrm{~ms}, 90^{\circ} \mathrm{C}$ \\
PLAC.E3 & B.2 & $t_{p \mathrm{H}_{2}}=2 \mathrm{~s}, t_{\mathrm{bypass}}=3 \mathrm{~s}$, variation of $t_{\mathrm{IN}, 1}$ and $t_{\mathrm{IN}, 2}, 90^{\circ} \mathrm{C}$ \\
PLAC.E4 & B.2/3, or no buffer & $t_{p \mathrm{H}_{2}}=2 \mathrm{~s}, t_{\text {bypass }}=3 \mathrm{~s}, t_{\mathrm{IN}, 1}=87.8 \mathrm{~ms}, t_{\mathrm{IN}, 2}=70.7 \mathrm{~ms}, 90^{\circ} \mathrm{C}$
\end{tabular}




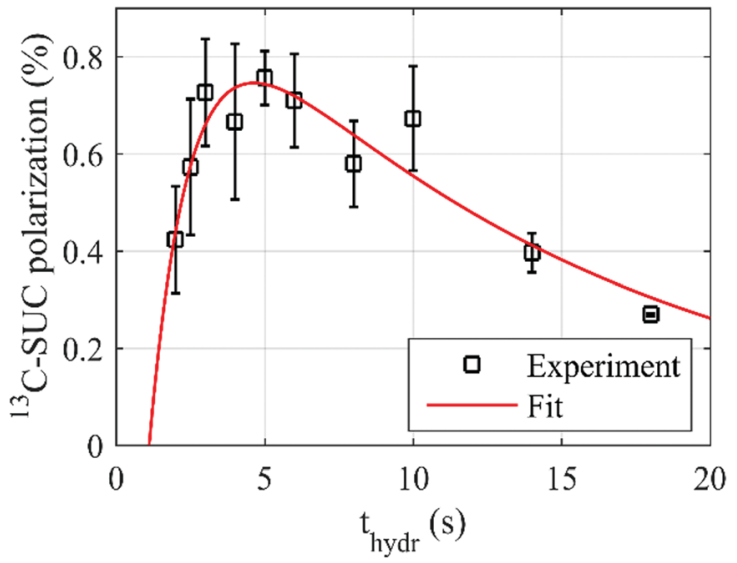

Fig. $4{ }^{13} \mathrm{C}$ polarization of SUC polarized with ECHO (squares) as a function of the hydrogenation duration $t_{\text {hydr }}$ and fit (line). FUM $(5 \mathrm{mM})$ was hydrogenated at $90{ }^{\circ} \mathrm{C}$ and 20 bar $\mathrm{pH}_{2}$ pressure for different durations ( $t_{\text {hydr }}$ ) before $\mathrm{ECHO}\left(t_{\mathrm{E}}=70.0 \mathrm{~ms}\right.$ ) was applied and the polarization was measured (SUC.E1). A function was fitted to the data to extract the hydrogenation and relaxation constant $t_{\text {cat }}$ and $t_{\text {spin }}$ (eqn (8)).

observed for $t_{\mathrm{E}}=130,150$, and $170 \mathrm{~ms}$ (Fig. 5 , red line and squares). When the isotope effect was taken into account $\left(\Delta_{\mathrm{CS}}=4 \mathrm{~Hz}\right)$, good agreement was found for all values of $t_{\mathrm{E}}$ (except for $t_{\mathrm{E}}=170 \mathrm{~ms}$ ) and the same overall scaling factor of 4.94 (Fig. 5, blue line and squares). An identical value of $\Delta_{\mathrm{CS}}$ was found by comparing experimental and simulated ${ }^{1} \mathrm{H}$ PASADENA spectra (see ESI $\dagger$ ).

Performance of the ADAPT sequence. The performance of the ADAPT sequence for hyperpolarizing SUC was investigated in simulations and experiments.

First, a simplified model was assumed, where decoupling was applied for the entire hydrogenation, neglecting reaction

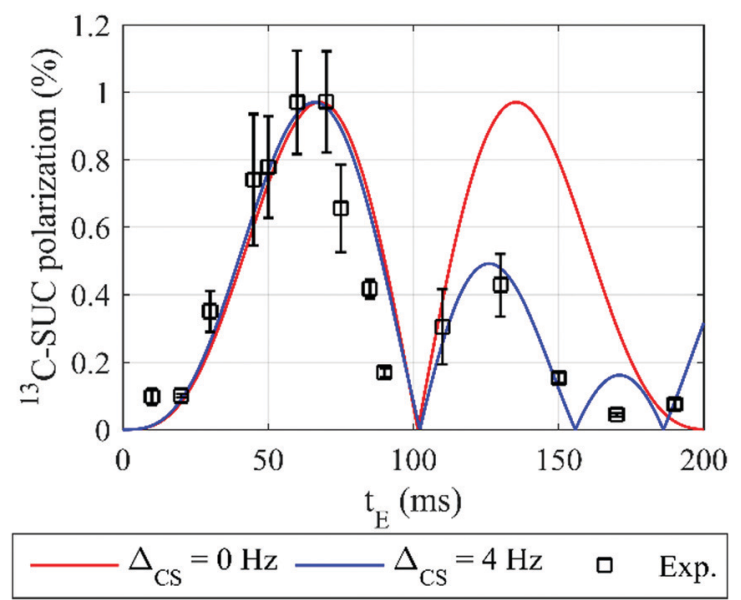

Fig. 5 Experimental (squares) and computed (lines) ${ }^{13} \mathrm{C}$ polarization of SUC as a function of $t_{E}$, the free evolution interval of the ECHO sequence. The polarization yield of SUC was simulated with and without the effect of the ${ }^{13} \mathrm{C}$ labeling on the ${ }^{1} \mathrm{H}$ chemical shift (red line: $\Delta_{\mathrm{CS}}=0 \mathrm{~Hz}$, blue line: $\Delta_{\mathrm{CS}}=$ $4 \mathrm{~Hz}$ ). The simulated polarization yield was scaled by a constant factor 4.9 to match the maximum of the experimental data (SUC.E2). The simulations matched the experiments better if chemically inequivalent protons were assumed (blue line). All data points were measured three times and the error bars represent the standard deviation. intermediates, and relaxation (eqn (6)). Here, a polarization of $P=$ $85.3 \%$ was obtained for $t_{\mathrm{AD}, 90^{\circ}}=33.63 \mathrm{~ms}$ and $\Delta_{\mathrm{CS}}=0 \mathrm{~Hz}$. By adding singlet-triplet mixing (eqn (7)), a significant decrease in ${ }^{13} \mathrm{C}$ polarization to $P=8.5 \%$ was observed $\left(P=5.7 \%\right.$ with $\mathrm{S}^{-} \mathrm{T}_{0}$ mixing and without decoupling).

Next, we investigated the hyperpolarization as a function of $t_{\mathrm{AD}, 90^{\circ}}$ (Fig. 6). Here, we found that the distribution of the polarization was shifted by $\approx 8 \mathrm{~ms}$ to smaller $t_{\mathrm{AD}, 90^{\circ}}$ if the isotope effect was considered $\left(\Delta_{\mathrm{CS}}=4 \mathrm{~Hz}\right.$ and decoupling on). The maximum polarization was $\approx 5 \%$ with $\mathrm{S}-\mathrm{T}_{0}$ mixing.

Experimentally, it was necessary to wait for $2 \mathrm{~s}$ after the injection of $p \mathrm{H}_{2}$ before the decoupling was tuned on to avoid inhomogeneous $B_{0}$ and ill - defined pulses because of the bubbles. ${ }^{44}$ This means that decoupling was switched off and on, resulting in two different density matrices. Using the results from the above experiments (eqn (8) and Fig. 4), we estimated that about $75 \%$ of FUM was hydrogenated in the first $2 \mathrm{~s}$ without decoupling, and $22 \%$ during the following $3 \mathrm{~s}$ with decoupling (resulting in $97 \%$ total hydrogenation in 5 s). We used these fractions and density matrices to simulate the expected total polarization as follows:

$$
P=\frac{0.754 \cdot P_{\text {NoDec. }}+0.216 \cdot P_{\text {Dec. }}}{0.97}
$$

These simulations yielded a similar distribution of the polarization as function of $t_{\mathrm{AD}, 90^{\circ}}$ as the simplified model, but with an

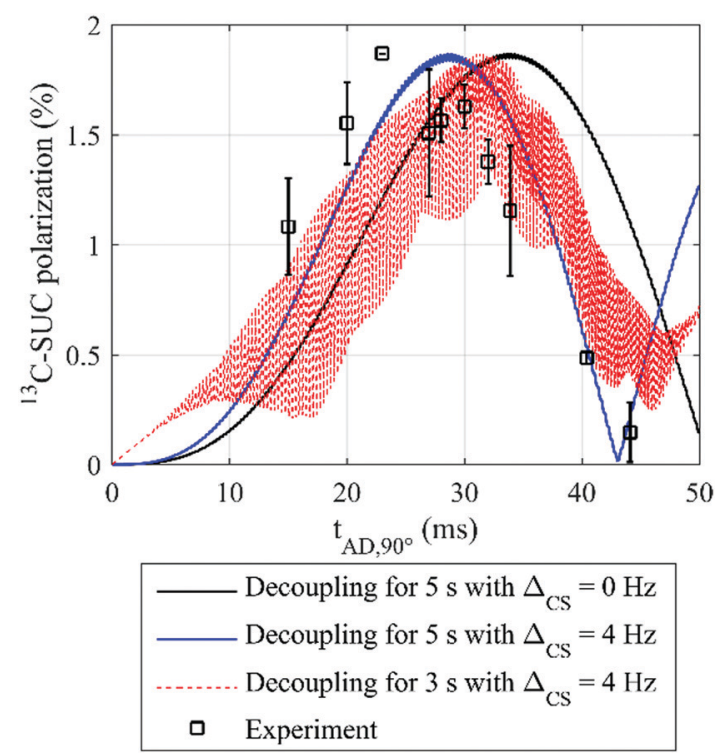

Fig. 6 Experimental and simulated (normalized) ${ }^{13} \mathrm{C}$ polarization of SUC using ADAPT. The polarization yield was simulated assuming decoupling during the entire hydrogenation reaction for chemically equivalent (black) and non-equivalent (blue) protons. Experimentally, decoupling was applied only for $3 \mathrm{~s}$ after a $2 \mathrm{~s}$ delay (squares). The corresponding simulations yielded an oscillating pattern (red). Experimentally, a maximum polarization of $\approx 1.9 \%$ was observed (squares), while the simulations yielded $\approx 5 \%$. All measured data points represent the mean of three measurements and the error bars correspond to the standard deviation (except for $t_{\mathrm{AD}, 90^{\circ}}=23.0 \mathrm{~ms}$ which was measured only once). All simulated polarization yields were scaled by an overall factor to match the experimental data (black, blue, and red lines). 
oscillation up to about $10 \%$ of the absolute value (Fig. 6 , red line). The maximum polarization was found to be $5.2 \%$ for $\approx 32 \mathrm{~ms}$.

Experimentally, a similar distribution of the polarization was found. The experiments were most similar to the simulations considering the isotope effect. The maximum polarization was about $1.9 \%$ at $t_{\mathrm{AD}, 90^{\circ}}=23.0 \mathrm{~ms}$ (SUC.E3, squares).

\section{Hyperpolarization of phospholactate}

SAMBADENA for PLAC was simulated with the SOT sequence PH-INEPT + . When the hydrogenation process was calculated numerically (eqn (3) and (4)), the resulting time-averaged 4-spin density matrix was given by pure longitudinal ${ }^{1} \mathrm{H}$ double spin order, i.e. by

$$
\overline{\rho_{0}}=\left[\frac{1}{4} \mathbb{E}-I_{1 z} I_{2 z}\right] \otimes \rho_{13} \mathrm{C} \otimes \rho_{31} \mathrm{P}
$$

to a good approximation. Based on this density matrix, simulations of the PH-INEPT+ sequence ex- or including the ${ }^{31} \mathrm{P}$ spin predicted a maximum ${ }^{13} \mathrm{C}$ polarization level of $P=41.2 \%$ for $t_{\mathrm{IN}, 1}=87.8 \mathrm{~ms}$ and $t_{\mathrm{IN}, 2}=70.7 \mathrm{~ms}$ in both cases.

Optimization of the hydrogenation duration. To obtain the optimal hydrogenation duration, we varied $t_{\text {hydr }}$ in the range of 1 to $30 \mathrm{~s}$. When using buffer B. 2 and $90{ }^{\circ} \mathrm{C}$ or $60{ }^{\circ} \mathrm{C}$ hydrogenation temperature, a maximum polarization of about $0.7 \%$ or $1.2 \%$, respectively, was found for $t_{\mathrm{hydr}}=5 \mathrm{~s}$ (PLAC.E1/2). For $90{ }^{\circ} \mathrm{C}$ reaction temperature, the lifetime of transferred spin order $t_{\text {spin }}$ and the hydrogenation constant $t_{\text {cat }}$ were determined as $(1.3 \pm 0.8) \mathrm{s}$ and $(17.9 \pm 8.8) \mathrm{s}$, respectively (eqn (8)), data not shown). Note that this is quite different to the values obtained for SUC. Fitting the two-site kinetic model to the MRI PLAC data suggested that only $(24 \pm 10) \%$ of all precursor molecules were hydrogenated after $t_{\text {hydr }}=5 \mathrm{~s}$ at $90{ }^{\circ} \mathrm{C}$ with a hydrogenation constant of $t_{\text {cat }}=(17.9 \pm 8.8) \mathrm{s}$. Assuming a $(24 \pm 10) \%$ hydrogenation yield, an effective ${ }^{13} \mathrm{C}$ polarization of $(4.2 \pm$ $0.4) \%$ was reached following Gaussian propagation of errors. This is 10 -fold less than predicted by simulations $(41.2 \%)$. A $63 \%$ hydrogenation yield was measured by high-resolution NMR spectroscopy of the same samples several hours after initializing the hydrogenation (see ESI $\dagger$ ). This indicates an ongoing hydrogenation after the hyperpolarization experiment and an effective polarization yield of about $1.6 \%$.

Variation of the free evolution intervals. The simulated parameters for PH-INEPT+ yielded a polarization yield of $P=$ $(0.63 \pm 0.11) \%$ for $t_{\mathrm{IN}, 1}=87.8 \mathrm{~ms}$ and $t_{\mathrm{IN}, 2}=70.7 \mathrm{~ms}$ using the sodium phosphate buffer B.2 at $90{ }^{\circ} \mathrm{C}$ (PLAC.E3, Fig. 7). By varying $t_{\mathrm{IN}, 1}$ and $t_{\mathrm{IN}, 2}$, we found that the experimental polarization increased slightly to $P=(0.82 \pm 0.11) \%$ when shorter intervals were used, i.e. $t_{\mathrm{IN}, 1}=60.0 \mathrm{~ms}$ and $t_{\mathrm{IN}, 2}=50.0 \mathrm{~ms}$. The measured ${ }^{13} \mathrm{C}$ polarizations were identical for $10 \mathrm{~ms} \leq t_{\mathrm{IN}, 2} \leq$ $110 \mathrm{~ms}$, except for $t_{\mathrm{IN}, 2}=50.0 \mathrm{~ms}$. However, the values were identical within two error intervals.

Please note that the measured polarization yield is about a factor of 65 smaller than predicted by computations. By comparing scaled simulated values and experimental data,
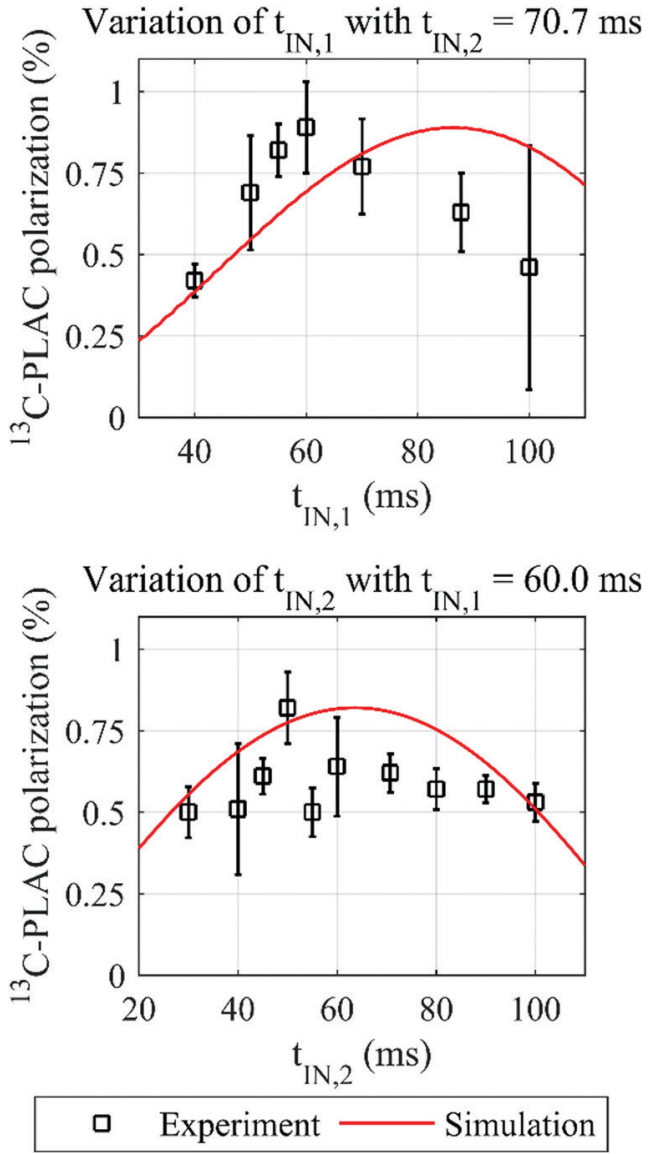

Fig. 7 Experimental and theoretical ${ }^{13} \mathrm{C}$-PLAC polarization as a function of the free evolution intervals of $\mathrm{PH}$-INEPT+. The intervals were empirically optimized (squares) and compared to simulations (red line). The computations did not suit the experimental outcome after scaling. The mean values and standard deviations are shown.

mismatches were found for the first and second evolution interval (Fig. 7).

Influence of $\mathbf{p H}$ buffer solution. The hyperpolarization experiments were repeated in differently buffered solutions with $t_{\mathrm{IN}, 1}=87.8 \mathrm{~ms}, t_{\mathrm{IN}, 2}=70.70 \mathrm{~ms}$, and a hydrogenation time of $t_{\text {hydr }}=5 \mathrm{~s}$ at $90{ }^{\circ} \mathrm{C}$ (PLAC.E4). When adjusting the $\mathrm{pH}$ value to 10.6 through the sodium phosphate buffer (B.2), a maximum polarization yield of $P=(0.62 \pm 0.11) \%$ was found. When a $\mathrm{pH}$ buffer based boric acid (B.3, pH 10.0) was used, a decreased polarization of $P=(0.17 \pm 0.15) \%$ was found. At $\mathrm{pH}$ of 3 before hydrogenation when no buffer solution was added to the solution, a mean polarization of $P=(0.16 \pm 0.05) \%$ was observed.

${ }^{1} \mathbf{H}$ and ${ }^{13} \mathbf{C}$ PLAC relaxation times. The relaxation times of thermally equilibrated PLAC in the B.2 solution at $\mathrm{pH} 10.6$, room temperature, and $7 \mathrm{~T}$ were determined as $T_{1}=(6.8 \pm 0.4) \mathrm{s}$ for the methyl and $T_{1}=(5.5 \pm 0.7) \mathrm{s}$ for methine proton after a hyperpolarization experiment. The $T_{2}$ relaxation time of the methyl proton at $1.25 \mathrm{ppm}$ was determined as $T_{2}=(3.5 \pm 0.2) \mathrm{s}$. $T_{1}=(31.0 \pm 1.0) \mathrm{s}$ and $T_{2} \approx 2 \mathrm{~s}$ were found for the ${ }^{13} \mathrm{C}$ nucleus. See $\mathrm{ESI} \dagger$ for more details. 


\section{Discussion}

In a previous SAMBADENA related publication, a fundamental hurdle for hyperpolarization of SUC, i.e. singlet-triplet mixing, has been identified. ${ }^{44}$ Here, the loss of polarization due to the ${ }^{13} \mathrm{C}$ isotope effect, poor hydrogenation, and fast $T_{1} / T_{2}$ relaxation is discussed for both of the SUC- and PLAC-molecules.

\section{Succinate}

The experimentally observed ${ }^{13} \mathrm{C}$ polarization yield of about $1 \%$ through the ECHO or ADAPT sequence was significantly lower than predicted by simulations considering singlet-triplet mixing, i.e. about 5\% for $\mathrm{ECHO}$ and $8 \%$ for ADAPT, respectively (Fig. 5 and 6). However, the simulated and measured dependencies on the free evolution intervals were in satisfying agreement when an overall scaling factor was introduced, when chemically inequivalent SUC-protons were hypothesized, and when the experimental workflow was considered. The scaling factor may be explained by $\approx 90 \% \mathrm{pH}_{2}$ enrichment and deviating flip angles within the sample volume. The ${ }^{13} \mathrm{C}$ isotope leads to a break of symmetry within the molecule, resulting in different Larmor frequencies of both protons. The assumed difference of $\Delta_{\mathrm{CS}}=$ $4 \mathrm{~Hz}$, i.e. a few $\mathrm{ppb}$, is in accordance with previously detected ${ }^{13} \mathrm{C}$-induced shifts of the ${ }^{1} \mathrm{H}$ Larmor frequency. ${ }^{58,59}$ Despite optimization of the free evolution intervals and hydrogenation duration, the herein reported polarization yields of $1 \%$ are substantially lower than those previously achieved through Goldman's sequence, i.e. $10 \% .{ }^{44}$ However, given the simplicity of ECHO, i.e. ${ }^{13} \mathrm{C}$ RF only and no decoupling, the sequence maybe beneficial for low-budget MR units.

\section{Phospholactate}

The experimental polarization yield of about $1 \%$ is lower than expected from computations where a ${ }^{13} \mathrm{C}$-PLAC polarization of roughly $41 \%$ was predicted (Fig. 7 ). ${ }^{1} \mathrm{H}-{ }^{31} \mathrm{P}$ and ${ }^{13} \mathrm{C}-{ }^{31} \mathrm{P} J$-coupling interaction did not affect the polarization yield in simulations.

Evidence for relaxation of spin order was found by variation of the intervals $t_{\mathrm{IN}, 1}$ and $t_{\mathrm{IN}, 2}$ as the experimental maximum was observed for shorter $t_{\mathrm{IN}, 1}$ intervals than predicted by simulations. Contrary, no prominent shift was observed by variation of the $t_{\mathrm{IN}, 2}$ interval. This finding is conclusive as the $t_{\mathrm{IN}, 2}$ interval of the PH-INEPT+ sequence serves for the transformation of ${ }^{13} \mathrm{C}$ out-phase in ${ }^{13} \mathrm{C}$ in-phase magnetization where only relaxation of ${ }^{13} \mathrm{C}$ is important. ${ }^{23}$

Please note that in previous studies, ${ }^{13} \mathrm{C}-\mathrm{HEP}$ was polarized to about $20 \%$ through the same SOT sequence at $7 \mathrm{~T}$ using a similar setup. ${ }^{42}$ Comparing the relaxation times and polarization levels of HEP and PLAC leads to the assumption that relaxation may partly explain the tremendous loss of ${ }^{13} \mathrm{C}$-PLAC polarization. First, the longitudinal relaxation times of PLAC, about $6 \mathrm{~s}$ for ${ }^{1} \mathrm{H}$ and $31 \mathrm{~s}$ for ${ }^{13} \mathrm{C}$, are a factor of 2 or 5 lower than for $\mathrm{HEP}^{56}\left(27 \mathrm{~s} \mathrm{for}{ }^{1} \mathrm{H}\right.$ and $75 \mathrm{~s}$ for ${ }^{13} \mathrm{C}$ in aqueous solution with the same catalyst). Second, the polarization transfer was about a factor of 17 more efficient in HEP than in PLAC. This follows by comparison of the simulated and measured $\mathrm{HEP}^{42}$ and PLAC polarization levels, i.e. $P_{\mathrm{HEP}, \mathrm{Sim}} \approx$ $49 \%, P_{\mathrm{HEP}, \mathrm{Exp}} \approx 20 \%, P_{\mathrm{PLAC}, \mathrm{Sim}} \approx 42 \%$, and $P_{\mathrm{PLAC}, \mathrm{Exp}} \approx 1 \%$.
Relaxation in PLAC is potentially more pronounced because of dipole-dipole interaction with the additional ${ }^{31} \mathrm{P}$ spin and by the adjacency of the lactate-OH group (Fig. 2). This hypothesis is supported by the fact that relaxation times depend on the field strength, temperature, and molecular environment. ${ }^{60}$ For instance, shorter $T_{1}$ relaxation times, i.e. $4.3 \mathrm{~s}$ for ${ }^{1} \mathrm{H}$ and $16.2 \mathrm{~s}$ for ${ }^{13} \mathrm{C}$, were measured at $9.4 \mathrm{~T}$ in earlier studies. ${ }^{34}$

The observed dependency of the polarization yield on reaction temperature and buffer solution might be caused by changes in the relaxation times and hydrogenation yields.

The experimental polarization yield of $1 \%$ is significantly lower than the $15 \%$ achieved in previous studies conducted at $5.75 \mathrm{mT}^{32}$ and related to the ${ }^{1} \mathrm{H}^{-13} \mathrm{C}$ transfer efficiency, to the hydrogenation reaction, and relaxation times. The achieved signal enhancement of about 1800 at $7 \mathrm{~T}$ is likely too low for interesting in vivo applications. Here, faster hydrogenation may prove advantageous to boost ${ }^{13} \mathrm{C}$ polarization yields to make this agent suitable for in vivo studies. Please note, that ions (due to the presence of a $\mathrm{pH}$ buffer) may lead to the cancellation of hyperpolarized signals as described in literature. ${ }^{61}$

\section{Future PHIP agents and their application in biomedical research}

Beside unsaturated double or triple bonds for permanent addition of $p \mathrm{H}_{2}$, additional requirements need to be fulfilled for the generation of PASADENA hyperpolarized ${ }^{13} \mathrm{C}$ agents for biomedical applications. First, chemically inequivalent protons are desirable to prevent polarization loss due to singlet-triplet mixing, challenges with decoupling sequences at high field, ${ }^{44}$ and potential isotope labeling effects. Second, molecules with relatively long ${ }^{1} \mathrm{H}$ and ${ }^{13} \mathrm{C}$ relaxation times are preferable to provide high ${ }^{13} \mathrm{C}$ polarization since the loss of spin coherence during hydrogenation and SOT is less dominant. The influence of relaxation during spin order transfer might be addressed by appropriate choice of the solvents and $\mathrm{pH}$ buffer solutions. Third, PASADENA agents with $\mathrm{OH}$-groups close to the $\mathrm{pH}_{2}$ nascnet protons and ${ }^{13} \mathrm{C}$ should be avoided to decrease the influence of spin exchange induced relaxation. When the requirements are (partly) fulfilled, ${ }^{13} \mathrm{C}$ polarization yields of metabolites at high field with pulsed SOT is feasible, e.g. $60 \%$ of ethyl acetate and $9 \%$ of pyruvate, respectively. ${ }^{22,41}$ SAMBADENA would allow to perform the hyperpolarization directly in the MRI, at low cost, and next to the application target reducing loss by relaxation.

\section{Conclusion and outlook}

In this contribution, the initial experience with the SAMBADENA hyperpolarization of PLAC, a derivate to obtain hyperpolarized $1{ }^{13} \mathrm{C}$-lactate, was shown. Moreover, we investigated the hyperpolarization of SUC by means of the ECHO and ADAPT sequences. The low polarizations about $1 \%$ were attributed to (a) the ${ }^{13} \mathrm{C}$ isotope effect, (b) low $T_{1} / T_{2}$ relaxation times, and (c) low hydrogenation yields. The symmetry of SUC is broken due to ${ }^{13} \mathrm{C}$ labeling (Fig. 1a). Consequently, the two SUC-protons 
precess with different Larmor frequencies $\left(\Delta_{\mathrm{CS}}=4 \mathrm{~Hz}\right)$ and the efficiency of spin order transfer is reduced. The value of $\Delta_{\mathrm{CS}}$ was obtained from simulations and experiments (Fig. 5 and ESI $\dagger$ ). In the case of PLAC, low relaxation times of the PLAC-protons and low hydrogenation yields may explain the loss of ${ }^{13} \mathrm{C}$ polarization. These results are of significance for hyperpolarization with SOT sequences at high magnetic fields. Higher hydrogenation yields and precursor molecule concentrations than in recent SAMBADENA experiments are achievable with a more sophisticated reactor design and experimental setup in future work.

\section{Conflicts of interest}

There are no conflicts to declare.

\section{Acknowledgements}

Funding support by the Forschungskommission (University Medical Center Freiburg, SCHM2146/20), the Emmy Noether Program of the DFG (HO 4604/2-1, 4604/2-2), the German Consortium for Translational Cancer Research (DKTK), the Cluster of Excellence "Precision Medicine in Inflammation" (PMI 1267), the RFBR and DFG (HO-4604/3-1, 19-53-12013), the research training group materials4brain (RTG 2154), the European Union's Horizon 2020 research and innovation programme under the Marie Sklodowska-Curie grant agreement No. 642773, and the Heinrich-Böll-Stiftung (ABS, P131623) is gratefully acknowledged. E. Y. C. thanks the National Science Foundation CHE-1904780, the National Cancer Institute 1R21CA220137, and the National Heart, Lung, and Blood Institute 1R21 HL154032.

\section{References}

1 T. Platt, et al., In vivo self-gated $23 \mathrm{Na}$ MRI at $7 \mathrm{~T}$ using an oval-shaped body resonator, Magn. Reson. Med., 2018, 80(3), 1005-1019.

2 A. M. Nagel, et al., In Vivo 35Cl MR Imaging in Humans: A Feasibility Study, Radiology, 2014, 271, 585-595.

3 S. C. Niesporek, et al., Reproducibility of $\mathrm{CMRO} 2$ determination using dynamic 17O MRI, Magn. Reson. Med., 2018, 79, 2923-2934.

4 R. Borowiak, J. Groebner, M. Haas, J. Hennig and M. Bock, Direct cerebral and cardiac 17O-MRI at 3 Tesla: initial results at natural abundance, Magn. Reson. Mater. Phys., Biol. Med., 2014, 27, 95-99.

5 K. V. Kovtunov, et al., Hyperpolarized NMR Spectroscopy: d-DNP, PHIP, and SABRE Techniques, Chem. - Asian J., 2018, 13, 1857-1871.

6 R. Aggarwal, D. B. Vigneron and J. Kurhanewicz, Hyperpolarized 1-[13C]-Pyruvate Magnetic Resonance Imaging Detects an Early Metabolic Response to Androgen Ablation Therapy in Prostate Cancer, Eur. Urol., 2017, 72, 1028-1029.

7 C. A. Müller, et al., Dynamic 2D and 3D mapping of hyperpolarized pyruvate to lactate conversion in vivo with efficient multi-echo balanced steady-state free precession at 3 T, NMR Biomed., 2020, 33, e4291.
8 C. Laustsen, et al., Hyperpolarized [1,4-13 C]fumarate imaging detects microvascular complications and hypoxia mediated cell death in diabetic nephropathy, Sci. Rep., 2020, 10, 1-10.

9 J. M. Park, et al., Hyperpolarized 13C NMR observation of lactate kinetics in skeletal muscle, J. Exp. Biol., 2015, 218, 3308-3318.

$10 \mathrm{~J} . \mathrm{H}$. Ardenkjaer-Larsen, et al., Increase in signal-to-noise ratio of $>10,000$ times in liquid-state NMR, Proc. Natl. Acad. Sci. U. S. A., 2003, 100, 10158-10163.

11 J. H. Ardenkjær-Larsen, et al., Cryogen-free dissolution dynamic nuclear polarization polarizer operating at $3.35 \mathrm{~T}$, 6.70 T, and 10.1 T, Magn. Reson. Med., 2019, 81, 2184-2194.

12 T. C. Eisenschmid, et al., Para hydrogen induced polarization in hydrogenation reactions, J. Am. Chem. Soc., 1987, 109, 8089-8091.

13 C. R. Bowers and D. P. Weitekamp, Parahydrogen and synthesis allow dramatically enhanced nuclear alignment, J. Am. Chem. Soc., 1987, 109, 5541-5542.

14 C. R. Bowers and D. P. Weitekamp, Transformation of Symmetrization Order to Nuclear-Spin Magnetization by Chemical Reaction and Nuclear Magnetic Resonance, Phys. Rev. Lett., 1986, 57, 2645.

15 S. Kadlecek, K. Emami, M. Ishii and R. Rizi, Optimal transfer of spin-order between a singlet nuclear pair and a heteronucleus, J. Magn. Reson., 2010, 205, 9-13.

16 C. Cai, A. M. Coffey, R. V. Shchepin, E. Y. Chekmenev and K. W. Waddell, Efficient Transformation of Parahydrogen Spin Order into Heteronuclear Magnetization, J. Phys. Chem. $B, 2013,117,1219-1224$.

17 M. Goldman, H. Jóhannesson, O. Axelsson and M. Karlsson, Design and implementation of 13C hyper polarization from para-hydrogen, for new MRI contrast agents, C. R. Chim., 2006, 9, 357-363.

18 V. A. Norton, Efficient generation of hyperpolarized molecules utilizing the scalar order of parahydrogen, California Institute of Technology, 2010.

19 G. Stevanato, Alternating Delays Achieve Polarization Transfer (ADAPT) to heteronuclei in PHIP experiments, J. Magn. Reson., 2017, 274, 148-162.

20 G. Stevanato, J. Eills, C. Bengs and G. Pileio, A pulse sequence for singlet to heteronuclear magnetization transfer: S2hM, J. Magn. Reson. San Diego Calif. 1997, 2017, 277, 169-178.

21 J. Eills, et al., Singlet order conversion and parahydrogeninduced hyperpolarization of $13 \mathrm{C}$ nuclei in near-equivalent spin systems, J. Magn. Reson., 2017, 274, 163-172.

22 S. Korchak, S. Yang, S. Mamone and S. Glöggler, Pulsed Magnetic Resonance to Signal-Enhance Metabolites within Seconds by utilizing para-Hydrogen, ChemistryOpen, 2018, 7, 344-348.

23 M. Haake, J. Natterer and J. Bargon, Efficient NMR Pulse Sequences to Transfer the Parahydrogen-Induced Polarization to Hetero Nuclei, J. Am. Chem. Soc., 1996, 118, 8688-8691.

24 S. Kadlecek, et al., A simple and low-cost device for generating hyperpolarized contrast agents using parahydrogen, NMR Biomed., 2011, 24, 933-942.

25 R. Borowiak, et al., A battery-driven, low-field NMR unit for thermally and hyperpolarized samples, Magn. Reson. Mater. Phys., Biol. Med., 2013, 26, 491-499. 
26 J.-B. Hövener, et al., Quality assurance of PASADENA hyperpolarization for 13C biomolecules, Magma, 2009, 22, 123-134.

27 J.-B. Hövener, et al., PASADENA hyperpolarization of 13C biomolecules: equipment design and installation, Magn. Reson. Mater. Phys., Biol. Med., 2009, 22, 111-121.

28 A. M. Coffey, et al., Open-Source Automated Parahydrogen Hyperpolarizer for Molecular Imaging Using 13C Metabolic Contrast Agents, Anal. Chem., 2016, 88, 8279-8288.

29 J. Agraz, A. Grunfeld, K. Cunningham, D. Li and S. Wagner, Improved PHIP polarization using a precision, low noise, voltage controlled current source, J. Magn. Reson., 2013, 235, 77-84.

30 J. Agraz, et al., LabVIEW-based control software for parahydrogen induced polarization instrumentation, Rev. Sci. Instrum., 2014, 85, 044705.

31 R. V. Shchepin, A. M. Coffey, K. W. Waddell and E. Y. Chekmenev, PASADENA Hyperpolarized 13C Phospholactate, J. Am. Chem. Soc., 2012, 134, 3957-3960.

32 R. V. Shchepin, A. M. Coffey, K. W. Waddell and E. Y. Chekmenev, Parahydrogen Induced Polarization of 113C-Phospholactate-d2 for Biomedical Imaging with $>$ 30,000,000-fold NMR Signal Enhancement in Water, Anal. Chem., 2014, 86, 5601-5605.

33 J.-B. Hövener, et al., Parahydrogen-Based Hyperpolarization for Biomedicine, Angew. Chem., Int. Ed., 2018, 57, 11140-11162.

34 O. G. Salnikov, et al., Effects of Deuteration of 13C-Enriched Phospholactate on Efficiency of Parahydrogen-Induced Polarization by Magnetic Field Cycling, J. Phys. Chem. C, 2018, 122, 24740-24749.

35 P. Bhattacharya, et al., Towards hyperpolarized 13Csuccinate imaging of brain cancer, J. Magn. Reson. San Diego Calif. 1997, 2007, 186, 150-155.

36 K. L. Billingsley, et al., Hyperpolarized [1,4-(13)C]diethylsuccinate: a potential DNP substrate for in vivo metabolic imaging, NMR Biomed., 2014, 27, 356-362.

37 E. Y. Chekmenev, et al., PASADENA Hyperpolarization of Succinic Acid for MRI and NMR Spectroscopy, J. Am. Chem. Soc., 2008, 130, 4212-4213.

38 Y. Zilberter, T. Zilberter and P. Bregestovski, Neuronal activity in vitro and the in vivo reality: the role of energy homeostasis, Trends Pharmacol. Sci., 2010, 31, 394-401.

39 A. P. Chen, et al., Feasibility of using hyperpolarized [1-13C]lactate as a substrate for in vivo metabolic 13C MRSI studies, Magn. Reson. Imaging, 2008, 26, 721-726.

$40 \mathrm{~J}$. Wang, et al., Dynamic $1 \mathrm{H}$ imaging of hyperpolarized [1-13C]lactate in vivo using a reverse INEPT experiment, Magn. Reson. Med., 2018, 79, 741-747.

41 S. Korchak, S. Mamone and S. Glöggler, Over 50\% 1H and 13C Polarization for Generating Hyperpolarized Metabolites-A para-Hydrogen Approach, ChemistryOpen, 2018, 7, 672-676.

42 A. B. Schmidt, et al., Liquid-state carbon-13 hyperpolarization generated in an MRI system for fast imaging, Nat. Commun., 2017, 8, ncomms14535.

43 A. B. Schmidt, et al., In vivo 13C-MRI using SAMBADENA, PLoS One, 2018, 13, e0200141.
44 S. Berner, et al., SAMBADENA Hyperpolarization of 13C-Succinate in an MRI: Singlet-Triplet Mixing Causes Polarization Loss, ChemistryOpen, 2019, 8, 728-736.

45 S. Bär, et al., On the spin order transfer from parahydrogen to another nucleus, J. Magn. Reson., 2012, 225, 25-35.

46 J. Barkemeyer, M. Haake and J. Bargon, Hetero-NMR Enhancement via Parahydrogen Labeling, J. Am. Chem. Soc., 1995, 117, 2927-2928.

47 R. E. Wasylishen and J. O. Friedrich, Deuterium isotope effects on the nitrogen chemical shift and $1 \mathrm{~J}(\mathrm{~N}, \mathrm{H})$ in the ammonium ion, J. Chem. Phys., 1984, 80, 585-587.

48 N. M. Zacharias, et al., Towards Real-time Metabolic Profiling of Cancer with Hyperpolarized Succinate, J. Mol. Imaging Dyn., 2016, 6, 1-9.

49 R. V. Shchepin, W. Pham and E. Y. Chekmenev, Dephosphorylation and biodistribution of 1-13C-phospholactate in vivo, J. Labelled Compd. Radiopharm., 2014, 57, 517-524.

50 F. Reineri, T. Boi and S. Aime, ParaHydrogen Induced Polarization of $13 \mathrm{C}$ carboxylate resonance in acetate and pyruvate, Nat. Commun., 2015, 6, 5858.

51 E. Cavallari, C. Carrera, S. Aime and F. Reineri, 13C MR Hyperpolarization of Lactate by Using ParaHydrogen and Metabolic Transformation in Vitro, Chem. - Eur. J., 2017, 23, 1200-1204.

52 P. E. Hansen, Isotope effects in nuclear shielding, Prog. Nucl. Magn. Reson. Spectrosc., 1988, 20, 207-255.

53 M. H. Levitt, R. Freeman and T. Frenkiel, Broadband heteronuclear decoupling, J. Magn. Reson. 1969, 1982, 47, 328-330.

54 P. Hübler, J. Natterer and J. Bargon, Indirect characterisation of hydrogenation intermediates using PASADENA NMR spectroscopy-evolution of zero-quantum coherence in $\mathrm{AB}$ spin systems, Ber. Bunsen-Ges., 1998, 102, 364-369.

55 J. Natterer, O. Schedletzky, J. Barkemeyer, J. Bargon and S. J. Glaser, Investigating Catalytic Processes with Parahydrogen: Evolution of Zero-Quantum Coherence in $\mathrm{AA}^{\prime} \mathrm{X}$ Spin Systems, J. Magn. Reson., 1998, 133, 92-97.

56 A. Schmidt, Liquid-state nuclear hyperpolarization without a polarizer: synthesis amid the magnet bore allows a dramatically enhanced nuclear alignment, Albert-Ludwigs-Universität Freiburg, 2020.

57 J.-B. Hövener, et al., A continuous-flow, high-throughput, high-pressure parahydrogen converter for hyperpolarization in a clinical setting, NMR Biomed., 2013, 26, 124-131.

58 M. Tiainen, H. Maaheimo, P. Soininen and R. Laatikainen, 13C isotope effects on $1 \mathrm{H}$ chemical shifts: NMR spectral analysis of 13C-labelled D-glucose and some 13C-labelled amino acids, Magn. Reson. Chem., 2010, 48, 117-122.

59 R. A. de Graaf, G. M. I. Chowdhury and K. L. Behar, Quantification of High-Resolution 1H-[13C] NMR Spectra from Rat Brain Extracts, Anal. Chem., 2014, 86, 5032-5038.

60 Wiley: Spin Dynamics: Basics of Nuclear Magnetic Resonance, 2nd Edition - Malcolm H. Levitt. http://eu.wiley.com/ WileyCDA/WileyTitle/productCd-0470511176.html.

61 A. Bröhl and R. Giernoth, PHIP NMR Spectroscopy in Ionic Liquids: Influence of Salts on the Intensity of Polarization Signals, Anal. Chem., 2014, 86, 10311-10314. 